\title{
Geometric characterization of hereditarily bijective Boolean networks
}

\author{
Paul Ruet \\ CNRS, Laboratoire Preuves, Programmes et Systèmes \\ Université Paris Diderot - Paris 7 \\ Case 7014, 75205 Paris Cedex 13, France \\ ruet@pps.univ-paris-diderot.fr
}

\begin{abstract}
The study of relationships between structure and dynamics of asynchronous Boolean networks has recently led to the introduction of hereditarily bijective maps and even or odd self-dual networks. We show here that these two notions can be simply characterized geometrically: through orthogonality between certain affine subspaces. We also use this characterization to provide a construction of the class of hereditarily bijective maps, and to study its stability properties.
\end{abstract}

\section{Introduction}

Boolean networks represent the dynamic interaction of components which can take two values, 0 and 1 . Introduced by von Neumann [17], they have been extensively used to model various biological networks, especially genetic regulatory networks, since the early works of S. Kauffman and R. Thomas $[5,6$, 13]. They are a discrete alternative to differential equations models, in which sufficently precise quantitative data often lack to accurately define the parameters. Regulatory interactions also exhibit strong thresholds effects (sigmoids), so that differential models are often conveniently approximated by piecewise linear equations [12], or one step further discretized into Boolean (or more generally multivalued) networks. See $[2,1]$ for recent surveys.

An increasingly active field of research is the study of asynchronous Boolean networks [15]. An asynchronous Boolean network with $n$ components may be presented by its phase space, which is a partial orientation of the lattice $\{0,1\}^{n}$, i.e., a directed graph whose vertex set is $\{0,1\}^{n}$ and whose edges only relate vertices which are 1-distant from each other for the Hamming distance. Asynchronous networks are thus nondeterministic dynamical systems, in which the value of at most one component may be updated at a time.

Although other update schemes of Boolean networks are studied as models of biological networks (in particular random [5] and synchronous networks [6, 3], as well as comparisons between update schemes [4]), asynchrony provides a simple mathematical framework in which all possible trajectories are considered. In the context of genetic networks, one may observe that trajectories of piecewise linear models almost surely (in the sense of measure theory) cross only one threshold 
hyperplane at a time, so that only the value of the corresponding component is updated in the discretized Boolean network, which thus follows the asynchronous update scheme.

The asymptotic dynamical properties of asynchronous Boolean networks (nature and number of attractors, e.g., existence and unicity of fixed points or attractive cycles) depend on their structure (the directed graph of interactions between components), but precise relationships between dynamics and structure are very difficult to characterize in general. In $[14,16]$, R. Thomas conjectures rules relating positive or negative cycles in the interaction graphs to non-unicity of fixed points (related to cellular differentiation) or sustained oscillations (related to homeostasis). It is possible to give a precise mathematical status to these rules in the framework of asynchronous networks, by identifying sustained oscillations with cyclic attractors and by defining local interaction graphs in a way similar to Jacobian matrices (Section 2 recalls the useful definitions). In this framework, while the positive rule is well understood [7], the rule relating (local) negative cycles to the existence of a cyclic attractor is unproved in general. In [9], the special case of and-or nets is partly solved, and in [10] the special case of antipodal attractive cycles for and-or nets is fully proved.

In the course of better understanding these relationships, two opposite notions have been independently introduced recently: even or odd self-dual networks in [8], and hereditarily bijective maps in [10]. They seem particularly relevant to the study of asynchronous Boolean networks which have a non trivial dynamics: indeed, the dynamics of a hereditarily bijective map is weakly terminating to a unique fixed point, hence particularly "simple", while even or odd self-dual subnetworks are necessary for the emergence of a "complex" dynamical behaviour.

In this article, we develop the theory of these classes of networks. We show that hereditary bijectivity, and hence even or odd self-duality, can be simply characterized geometrically: through orthogonality between certain affine subspaces (Section 4). We use this characterization to provide a construction of the class of hereditarily bijective maps (Section 5), and to study its stability properties, in particular to prove that it is stable under inverse. We also study the relationship between the invertibility of a Boolean map and of its Jacobian matrices: we show that if a map from $\{0,1\}^{n}$ to itself has all its Jacobian matrices hereditarily invertible, then it is hereditarily bijective (while the same statement without heredity is known to be false).

\section{Asynchronous Boolean networks}

We need some preliminary definitions and notations. $\mathbb{B}$ denotes the set $\{0,1\}$. Boolean sum $(+)$ and product $(\cdot)$ equip $\mathbb{B}$ with the structure of the field $\mathbb{F}_{2}$.

Let $\left\{e_{1}, \ldots, e_{n}\right\}$ be the canonical basis of the vector space $\mathbb{B}^{n}$, and for each $I \subseteq\{1, \ldots, n\}, e_{I}=\sum_{i \in I} e_{i}$. For $x, y \in \mathbb{B}^{n}, v(x, y)$ denotes the subset $I \subseteq$ $\{1, \ldots, n\}$ such that $x+y=e_{I}$, and the Hamming distance $d(x, y)$ is defined as the cardinality of $v(x, y)$. 


$$
\begin{aligned}
& f_{1}(x)=\left(x_{2}+1\right) x_{3} \\
& f_{2}(x)=\left(x_{3}+1\right) x_{1} \\
& f_{3}(x)=\left(x_{1}+1\right) x_{2}
\end{aligned}
$$

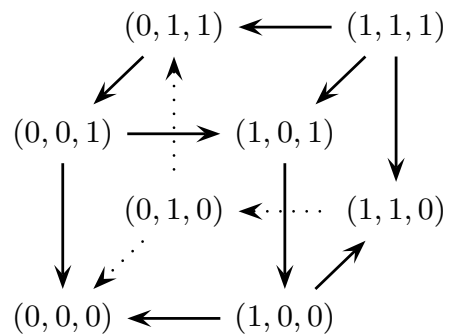

Fig. 1. A Boolean map $f: \mathbb{B}^{3} \rightarrow \mathbb{B}^{3}$ and the asynchronous dynamics $\Gamma(f)$ associated to it. For instance, the point $x=(1,0,0)$ in $\Gamma(f)$ has two outgoing edges to $x+e_{1}=$ $(0,0,0)$ and $x+e_{2}=(1,1,0)$ because $f(x)=(0,1,0)=x+e_{1}+e_{2}$.

Asynchronous Boolean networks can be equivalently presented in terms of directed graphs or in terms of Boolean maps. An asynchronous Boolean network can be defined:

1. either as a directed graph whose vertex set is $\mathbb{B}^{n}$ and whose edges only relate vertices which are 1-distant from each other (for any edge from $x$ to $y, d(x, y)=1)$;

2. or as a map from $\mathbb{B}^{n}$ to $\mathbb{B}^{n}$.

The two presentations indeed carry the same information (see Figure 1):

1. To a directed graph $\gamma$ as above, we may associate a map $\Phi(\gamma): \mathbb{B}^{n} \rightarrow \mathbb{B}^{n}$ by $\Phi(\gamma)(x)=x+e_{I}$, where $\left\{\left(x, x+e_{i}\right), i \in I\right\}$ is the set of edges going from $x$ in $\gamma$.

2. Conversely, given a map $f: \mathbb{B}^{n} \rightarrow \mathbb{B}^{n}$ we may define a directed graph $\Gamma(f)$ with vertex set $\mathbb{B}^{n}$ and an edge from $x$ to $y$ when for some $i, y=x+e_{i}$ and $f_{i}(x) \neq x_{i}$. Here, $f_{i}$ is defined by $f_{i}(x)=f(x)_{i}$. In that case, $d(x, y)=1$, and clearly, $\Gamma$ and $\Phi$ are inverses of each other.

We call $\Gamma(f)$ the asynchronous dynamics associated to $f$. As we shall consider asynchronous Boolean networks as dynamical systems, the coordinates $i$ such that $f_{i}(x) \neq x_{i}$ may naturally be considered as the degrees of freedom of $x$.

\subsection{Dynamical properties}

We shall be interested in the following asymptotic dynamical properties of asynchronous Boolean networks.

Let $f: \mathbb{B}^{n} \rightarrow \mathbb{B}^{n}$. A trajectory is a path in $\Gamma(f)$, and an attractor a terminal strongly connected component of $\Gamma(f)$. An attractor which is not a singleton (i.e., which does not consist in a fixed point) is called a cyclic attractor. Attractive cycles, i.e., cyclic trajectories $\theta$ such that for each point $x \in \theta, d(x, f(x))=1$, are examples of cyclic attractors. Observe that attractive cycles are deterministic, since any point in $\theta$ has a unique degree of freedom. 
Recall that $f$ is weakly terminating when for any $x \in \mathbb{B}^{n}$, some trajectory leaving $x$ leads to a fixed point. Therefore, $f$ has a cyclic attractor if and only if it is not weakly terminating. A stronger form of weak termination may be defined as follows. Given $f: \mathbb{B}^{n} \rightarrow \mathbb{B}^{n}$, a path from $x \in \mathbb{B}^{n}$ to $y \in \mathbb{B}^{n}$ in $\Gamma(f)$ is called a direct trajectory when its length is minimal, i.e., equals $d(x, y)$. And $\Gamma(f)$ is said to be directly terminating when for any point $x \in \mathbb{B}^{n}$ there exists a direct trajectory from $x$ to some fixed point.

For instance, the network $f$ defined in Figure 1 has a (non-attractive) cyclic trajectory $(1,0,0) \rightarrow(1,1,0) \rightarrow(0,1,0) \rightarrow(0,1,1) \rightarrow(0,0,1) \rightarrow(1,0,1) \rightarrow$ $(1,0,0)$, but it is directly terminating to a unique fixed point. We shall see in Section 3 that this property of direct termination is actually a consequence of the fact that $f+$ id is hereditarily bijective (Theorem 3 ).

\subsection{Subcubes and subnetworks}

Given $x \in \mathbb{B}^{n}$ and $I \subseteq\{1, \ldots, n\}$, the subset $x[I]$ consists in all points $y$ such that $y_{i}=x_{i}$ for each $i \notin I$; subsets of the form $x[I]$ are called $I$-subcubes, or simply subcubes of $\mathbb{B}^{n}$. If $y=x+e_{I}$, the subcube $x[I]$ is also denoted by $[x, y]$.

Subcubes of $\mathbb{B}^{n}$ are affine subspaces: indeed, the vector space $0[I]=\left\{e_{J} \mid J \subseteq\right.$ $I\}$ acts faithfully and transitively on $x[I]$. However, not every affine subspace is a subcube: the subset $\{(0,0),(1,1)\}$ is an affine subspace because $(1,1)+(1,1)=$ $(0,0)$, but it is clearly not a subcube.

For any subcube $\kappa$, let $\pi_{\kappa}: \mathbb{B}^{n} \rightarrow \kappa$ be the projection onto $\kappa$, defined as follows: if $\kappa=x[I]$,

$$
\left(\pi_{\kappa}(y)\right)_{i}= \begin{cases}y_{i} & \text { if } i \in I \\ x_{i} & \text { otherwise }\end{cases}
$$

Let also $\iota_{\kappa}: \kappa \rightarrow \mathbb{B}^{n}$ be the inclusion map. It is then immediate that $\pi_{\kappa} \circ \iota_{\kappa}$ is the identity. For any $f: \mathbb{B}^{n} \rightarrow \mathbb{B}^{n}$, let

$$
f \uparrow_{\kappa}=\pi_{\kappa} \circ f \circ \iota_{\kappa}: \kappa \rightarrow \kappa
$$

A subnetwork of $f$ is a map $f \uparrow_{\kappa}$ for some subcube $\kappa$. The asynchronous dynamics $\Gamma\left(f \uparrow_{\kappa}\right)$ is easily shown to be the subgraph of $\Gamma(f)$ induced by vertices in $\kappa$, a characterization which may be taken as an alternative, more intuitive, definition of subnetworks.

We shall need the following lemmas.

Lemma 1. The projection onto any I-subcube is an affine map, with associated linear transformation the projection $\pi_{0[I]}$ from $\mathbb{B}^{n}$ onto the linear subspace $0[I]$.

Proof. If $\kappa$ is an $I$-subcube, then:

$$
\pi_{\kappa}(x)=\pi_{\kappa}(0)+\pi_{0[I]}(x) .
$$

Indeed, for any $i \notin I,\left(\pi_{\kappa}(x)\right)_{i}=\left(\pi_{\kappa}(0)\right)_{i}$ and $\left(\pi_{0[I]}(x)\right)_{i}=0$. And for any $i \in I$, $\left(\pi_{\kappa}(x)\right)_{i}=\left(\pi_{0[I]}(x)\right)_{i}=x_{i}$ and $\left(\pi_{\kappa}(0)\right)_{i}=0$. 
Lemma 2. Let $\kappa=x[I]$ and $\lambda=y[J]$ be any two subcubes of $\mathbb{B}^{n}$. The image of $\lambda$ under $\pi_{\kappa}$ is the subcube $\pi_{\kappa}(y)[I \cap J]$. In other terms, $\pi_{\kappa}(y[J])=\pi_{\kappa}(y)[J] \cap \kappa$.

Proof. A point of $\lambda$ is of the form $y+e_{K}$ for some $K \subseteq J$. By Equation (1), for any $K \subseteq J$ :

$$
\pi_{\kappa}\left(y+e_{K}\right)=\pi_{\kappa}(y)+\pi_{0[I]}\left(e_{K}\right)=\pi_{\kappa}(y)+e_{I \cap K}
$$

When $K$ varies among all subsets of $J, I \cap K$ varies among all subsets of $I \cap J$. Therefore the image of $\lambda$ under $\pi_{\kappa}$ equals $\pi_{\kappa}(y)[I \cap J]$.

Now, observe that $z[I \cap J]=z[I] \cap z[J]$ for any $z$. Since $\pi_{\kappa}(y)[I]=x[I]=\kappa$, we conclude that $\pi_{\kappa}(y)[I \cap J]=\pi_{\kappa}(y)[J] \cap \kappa$.

Corollary 1. If $x, y \in \mathbb{B}^{n}$ and $\kappa$ is any subcube, then $\pi_{\kappa}([x, y])=\left[\pi_{\kappa}(x), \pi_{\kappa}(y)\right]$.

\section{Hereditarily bijective maps and even or odd self-dual networks}

A map $f: \mathbb{B}^{n} \rightarrow \mathbb{B}^{n}$ is said to be hereditarily bijective (resp. hereditarily ufp) [10] when for any subcube $\kappa, f \uparrow_{\kappa}$ is bijective (resp. has a unique fixed point). A pair $(x, y) \in \mathbb{B}^{n} \times \mathbb{B}^{n}$ called a mirror pair of $f: \mathbb{B}^{n} \rightarrow \mathbb{B}^{n}$ when $(f+\mathrm{id}) \uparrow_{[x, y]}(x)=$ $(f+\mathrm{id}) \uparrow_{[x, y]}(y)$, i.e., when $x$ and $y$ have the same degrees of freedom for the projected map $f \uparrow_{[x, y]}$.

For any $x \in \mathbb{B}^{n}$, the translation $t_{x}$ maps $y \in \mathbb{B}^{n}$ to $x+y$. The following proposition establishes some immediate stability properties of the class of hereditarily bijective maps.

Proposition 1. The class of hereditarily bijective maps is stable under:

1. composition with translations: if $f: \mathbb{B}^{n} \rightarrow \mathbb{B}^{n}$ is hereditarily bijective and $x, y \in \mathbb{B}^{n}$, then so is $t_{x} \circ f \circ t_{y}$;

2. permutation of coordinates: if $f: \mathbb{B}^{n} \rightarrow \mathbb{B}^{n}$ is hereditarily bijective and $\sigma \in \mathfrak{S}_{n}$, then so is $f^{\sigma}=\sigma \circ f \circ \sigma^{-1}$, where $\sigma$ acts on $\mathbb{B}^{n}$ by permuting coordinates.

Proof. The first property follows from the fact that translations are (hereditarily) bijective and stable under projection on subcubes. The second property follows from the fact that $f^{\sigma} \uparrow_{\kappa}=\left(f \uparrow_{\sigma(\kappa)}\right)^{\sigma}$.

We shall use the results of Section 4 to give another stability property in Corollary 3. Let now id denote the identity map from $\mathbb{B}^{n}$ to itself. The following theorem relates the above three definitions.

Theorem 1 (Ruet [10]). For any $f: \mathbb{B}^{n} \rightarrow \mathbb{B}^{n}$, the following are equivalent:

1. $f+\mathrm{id}$ is hereditarily bijective;

2. $f$ is hereditarily ufp;

3. $f$ has no mirror pair. 




Fig. 2. A network $g: \mathbb{B}^{3} \rightarrow \mathbb{B}^{3}$ such that $g+$ id is not (hereditarily) bijective.

A point $x \in \mathbb{B}^{n}$ is said to be even (resp. odd) [8] when $\sum_{i=1}^{n} x_{i}=0$ (resp. 1). The sum here is again addition in the field $\mathbb{F}_{2}$. A map $f: \mathbb{B}^{n} \rightarrow \mathbb{B}^{n}$ is even (resp. $o d d$ ) when the image of $f+$ id is the set of even (resp. odd) points of $\mathbb{B}^{n}$. Let $\bar{x}$ denote the antipode $x+e_{1}+\cdots+e_{n}$ of $x \in \mathbb{B}^{n}$. Now, a map $f$ is self-dual when for any $x \in \mathbb{B}^{n}, f(\bar{x})=\overline{f(x)}$, i.e., $(x, \bar{x})$ is a mirror pair.

Point 2 of the following theorem essentially asserts that if $f+$ id is not hereditarily bijective, not only has $f$ a mirror pair, but it has an even or odd self-dual subnetwork.

Theorem 2 (Richard [8]). Let $f: \mathbb{B}^{n} \rightarrow \mathbb{B}^{n}$.

1. If for any $i \in\{1, \ldots, n\}$ and $\{1, \ldots, n\} \backslash\{i\}$-subcube $\kappa,(f+\mathrm{id}) \uparrow_{\kappa}$ is bijective, and if $f+\mathrm{id}$ is not bijective, then $f$ is even or odd, and self-dual.

2. $f+\mathrm{id}$ is hereditarily bijective if and only if $f$ has no even or odd self-dual subnetwork.

The asynchronous dynamics of hereditarily bijective maps can then be characterized as follows.

Theorem 3 (Richard [8], Ruet [10]). If $f+\mathrm{id}$ is hereditarily bijective, then $\Gamma(f)$ has a unique attractor, this attractor is a fixed point and $\Gamma(f)$ is directly terminating (in particular, it is weakly terminating).

As we have already observed, for the network $f$ of Figure $1, f+$ id is hereditarily bijective and indeed, $f$ is directly terminating to a unique fixed point. On the other hand, flipping the arrow from $(0,1,0)$ to $(0,0,0)$ in $f$ gives rise to the network $g$ of Figure 2 without fixed point: $g+$ id is not bijective (hence not hereditarily bijective) as it never takes value 0 , and for $\kappa=(0,0,0)[2,3]=$ $[(0,0,0),(0,1,1)], g \uparrow_{\kappa}$ is an odd self-dual subnetwork.

\subsection{Dynamics and structure}

The above definitions are used in $[8,10]$ to understand the relationships between the dynamics of an asynchronous Boolean network $f$ and the structure of its Jacobian matrices $\mathscr{J}(f)(x)$ and interaction graphs $\mathscr{G}(f)(x)$. Let us recall the definitions of $\mathscr{J}(f)(x)$ and $\mathscr{G}(f)(x)$. 
Given $\varphi: \mathbb{B}^{n} \rightarrow \mathbb{B}$ and $i \in\{1, \ldots, n\}$, the discrete $i^{\text {th }}$ partial derivative $\partial \varphi / \partial x_{i}=\partial_{i} \varphi: \mathbb{B}^{n} \rightarrow \mathbb{B}$ maps each $x \in \mathbb{B}^{n}$ to

$$
\partial_{i} \varphi(x)=\varphi(x)+\varphi\left(x+e_{i}\right) .
$$

The + here is the addition of the field $\mathbb{F}_{2}$, therefore $\partial_{i} \varphi(x)=1$ if and only if $\varphi(x) \neq \varphi\left(x+e_{i}\right)$. Now, given $f: \mathbb{B}^{n} \rightarrow \mathbb{B}^{n}$ and $x \in \mathbb{B}^{n}$, the discrete Jacobian matrix $\mathscr{J}(f)(x)$ is the $n \times n$ matrix with entries $\mathscr{J}(f)(x)_{i, j}=\partial_{j} f_{i}(x)$. And $\mathscr{G}(f)(x)$, the interaction graph of $f$ at $x$, is defined [11] to be the directed graph with vertex set $\{1, \ldots, n\}$ and an edge from $j$ to $i$ when $\mathscr{J}(f)(x)_{i, j}=1$. The Jacobian matrix $\mathscr{J}(f)(x)$ is therefore simply the transpose of the adjacency matrix of $\mathscr{G}(f)(x)$, and $\mathscr{G}(f)(x)$ is the graph underlying the signed interaction graph defined in [7].

In $[8,10]$, a theorem of Shih and Dong on unicity of fixed points is improved as follows.

Theorem 4. Let $f: \mathbb{B}^{n} \rightarrow \mathbb{B}^{n}$.

1. If for any $x \in \mathbb{B}^{n}, \mathscr{G}(f)(x)$ has no cycle, then $f$ has a unique fixed point (Shih and Dong [11]).

2. If $f+\mathrm{id}$ is not hereditarily bijective, then there exist two points $x, y \in \mathbb{B}^{n}$ such that $\mathscr{G}(f)(x)$ and $\mathscr{G}(f)(y)$ have a cycle (Ruet [10]).

3. If $f+\mathrm{id}$ is not bijective, then for some $k \in\{1, \ldots, n\}$, there exist $2^{k}$ points $x \in \mathbb{B}^{n}$ such that $\mathscr{G}(f)(x)$ has a cycle of length at most $k$ (Richard [8]).

In [10], it is also shown that the invertibility of all Jacobian matrices $\mathscr{J}(f)(x)$ does not entail invertibility of $f: \mathbb{B}^{n} \rightarrow \mathbb{B}^{n}$. We prove here that the stronger condition of hereditary invertibility does.

Definition 1 (Hereditary invertibility, nilpotence). An $n \times n$ matrix with entries in $\mathbb{B}$ is said to be hereditarily invertible (resp. hereditarily nilpotent) when all its principal minors are.

Lemma 3. Let $M$ be an $n \times n$ matrix with entries in $\mathbb{B}$. The following are equivalent:

1. the graph associated to $M$ has no cycle;

2. $M$ is hereditarily nilpotent;

3. $\mathscr{I}+M$ is hereditarily invertible, where $\mathscr{I}$ denotes the identity matrix.

Proof. It is proved in [10] that (1) implies $M$ is nilpotent, hence hereditarily nilpotent because induced subgraphs of $M$ have no cycle either. Therefore, (1) implies (2). Clearly, (2) implies (3).

It remains to prove that (3) implies (1). If $\mathscr{I}+M$ is hereditarily invertible, its diagonal is necessarily $(1, \ldots, 1)$. Assume for a contradiction that the graph associated to $M$ has a cycle: let $C$ be a minimal cycle, i.e., one without chord, and $|C|$ be its vertex set. Then the principal minor $(\mathscr{I}+M)_{|C|}$ is the sum of $\mathscr{I}_{|C|}$ with the matrix of a cyclic permutation: it is therefore not invertible, contradicting the hypothesis. 
Theorem 5. Let $f: \mathbb{B}^{n} \rightarrow \mathbb{B}^{n}$. If $\mathscr{J}(f)(x)$ is hereditarily invertible for each $x \in \mathbb{B}^{n}$, then $f$ is hereditarily bijective.

Proof. By Lemma 3 , the condition of the theorem implies that, for any $x \in \mathbb{B}^{n}$, $\mathscr{I}+\mathscr{J}(f)(x)=\mathscr{J}(f+\mathrm{id})(x)$ has no cycle. By Theorem $4, f$ is then hereditarily bijective.

\section{Hereditary bijectivity and orthogonality}

We now turn to the main topic of this paper. A symmetric and nondegenerate bilinear form $\langle\cdot, \cdot\rangle: \mathbb{B}^{n} \times \mathbb{B}^{n} \rightarrow \mathbb{B}$ is defined on the vector space $\mathbb{B}^{n}=\mathbb{F}_{2}^{n}$ by $\langle x, y\rangle=\sum_{i=1}^{n} x_{i} y_{i}$, with sum and product in $\mathbb{F}_{2}$. As usual, two vectors $x, y$ are orthogonal when $\langle x, y\rangle=0$, a symmetric relation denoted by $x \perp y$. Let $A$ and $B$ be two affine subspaces of $\mathbb{B}^{n}$, with underlying vector spaces $V$ and $W$ respectively: $A$ and $B$ are said to be orthogonal (denoted by $A \perp B$ ) when for any two vectors $v \in V$ and $w \in W, v \perp w$.

We shall use orthogonality of subcubes to characterize hereditary bijectivity.

Theorem 6. For any $f: \mathbb{B}^{n} \rightarrow \mathbb{B}^{n}, f$ is hereditarily bijective if and only if for any $x, y \in \mathbb{B}^{n}$ such that $x \neq y,[x, y] \not \perp[f(x), f(y)]$.

We first need the following lemmas.

Lemma 4. Two subcubes $x[I], y[J]$ of $\mathbb{B}^{n}$ are orthogonal if and only if $I \cap J=\varnothing$.

Proof. The vector spaces underlying $x[I]$ and $y[J]$ are spanned by the sets $\left\{e_{i} \mid i \in\right.$ $I\}$ and $\left\{e_{j} \mid j \in J\right\}$ respectively. Therefore $x[I]$ and $y[J]$ are orthogonal if and only if any two spanning vectors $e_{i}, e_{j}$, with $(i, j) \in I \times J$, are orthogonal. As $\left\langle e_{i}, e_{j}\right\rangle=\delta_{i, j}$, this happens exactely when $i \neq j$ for any $(i, j) \in I \times J$, i.e., $I \cap J=\varnothing$.

Lemma 5. Projections onto subcubes are orthogonal projections.

Proof. By Lemma 1, it suffices to prove that the linear projection onto any linear subspace $0[I]$, with $I \subseteq\{1, \ldots, n\}$, is an orthogonal projection. The null space of $\pi_{0[I]}$ is clearly the subspace $0[J]$, where $J=\{1, \ldots, n\} \backslash I$. By Lemma 4 , we may then conclude that the null space $0[J]$ and the range $0[I]$ of $\pi_{0[I]}$ are orthogonal, as expected.

We now turn to the proof of Theorem 6 .

Let us first prove that if $f$ is hereditarily bijective, then for any $x, y \in \mathbb{B}^{n}$ such that $x \neq y,[x, y] \not \perp[f(x), f(y)]$. Assume for a contradiction that for some $x \neq y$, $[x, y] \perp[f(x), f(y)]$. Let $\kappa=[x, y]$ and $\lambda=[f(x), f(y)]$, so that $\kappa \perp \lambda$. By Lemma $5, \pi_{\kappa}$ maps the whole subcube $\lambda$ to a single point. Hence in particular, the two points $f(x)$ and $f(y)$ are mapped by $\pi_{\kappa}$ to the same point $\pi_{\kappa}(f(x))=\pi_{\kappa}(f(y))$. Therefore the two points $x, y \in \kappa$ are mapped by $f \uparrow_{\kappa}=\pi_{\kappa} \circ f \circ \iota_{\kappa}$ to the same point, and $f \uparrow_{\kappa}$ is not bijective: contradiction. 
Conversely, if $f$ is not hereditarily bijective, then $f \uparrow_{\kappa}$ is not bijective for some subcube $\kappa$ : there exist $x, y \in \kappa$ such that $x \neq y$ and $f \uparrow_{\kappa}(x)=f \uparrow_{\kappa}(y)$. In particular, we have:

$$
\pi_{[x, y]}(f(x))=f \uparrow_{[x, y]}(x)=f \uparrow_{[x, y]}(y)=\pi_{[x, y]}(f(y)),
$$

and $\pi_{[x, y]}$ maps $f(x)$ and $f(y)$ to the same point. By Corollary $1, \pi_{[x, y]}$ thus maps the subcube $[f(x), f(y)]$ to a single point. This implies that $[x, y] \perp[f(x), f(y)]$ by Lemma 5 . This completes the proof of Theorem 6 , and we may summarize the above characterizations as follows.

Corollary 2. The following are equivalent:

1. $f+\mathrm{id}$ is hereditarily bijective;

2. $f$ is hereditarily ufp;

3. $f$ has no mirror pair;

4. $f$ has no even or odd self-dual subnetwork;

5. for any $x \neq y,[x, y] \not \perp[f(x)+x, f(y)+y]$.

Another consequence of Theorem 6 is the following stability property of the class of hereditarily bijective maps.

Corollary 3. Inverses of hereditarily bijective maps are hereditarily bijective.

Proof. By Theorem 6, we have to prove that if $f$ is hereditarily bijective, then for any $x \neq y,[x, y] \not \perp\left[f^{-1}(x), f^{-1}(y)\right]$. When $x \neq y, f^{-1}(x) \neq f^{-1}(y)$, hence, again by Theorem $6,\left[f^{-1}(x), f^{-1}(y)\right] \perp\left[f\left(f^{-1}(x)\right), f\left(f^{-1}(y)\right)\right]=[x, y]$.

This property is especially interesting in view of the fact that hereditarily bijective maps do not form a category: $f:\left(x_{1}, x_{2}\right) \mapsto\left(x_{1}, x_{1}+x_{2}\right)$ and $g:\left(x_{1}, x_{2}\right) \mapsto$ $\left(x_{1}+x_{2}, x_{2}\right)$ are hereditarily bijective, but their composite $g \circ f:\left(x_{1}, x_{2}\right) \mapsto$ $\left(x_{2}, x_{1}+x_{2}\right)$ is not.

\section{Constructions of even or odd self-dual networks and hereditarily bijective maps}

Even self-dual networks may be constructed in full generality as follows. Given $\sigma \in \mathfrak{S}_{n}$, let $f: \mathbb{B}^{n+1} \rightarrow \mathbb{B}^{n+1}$ be the self-dual network defined on the subcube $0[\{1, \ldots, n\}]$ by $f(x, 0)=(\sigma(x), 0)$ if $\sigma(x)$ is even, $(\sigma(x), 1)$ otherwise (and selfduality determines $f(x, 1))$ : then $f$ is clearly even, and any even self-dual network can be constructed in this way. Replacing even by odd in the above definition provides arbitrary odd self-dual networks.

Constructing all hereditarily bijective maps is less immediate. Starting from hereditarily bijective maps $\sigma, \tau: \mathbb{B}^{n} \rightarrow \mathbb{B}^{n}$, let $f: \mathbb{B}^{n+1} \rightarrow \mathbb{B}^{n+1}$ be defined by $f(x, 0)=(\sigma(x), 0)$ and $f(x, 1)=(\tau(x), 1)$. Then $f$ is clearly hereditarily bijective. But in general, there exist other hereditarily bijective maps projecting to $\sigma$ and $\tau$. Let $S$ be a subset of $\mathbb{B}^{n}$ which is stable under the action of $\tau^{-1} \sigma$, and let $g$ be defined by $f(x, 0)=(\sigma(x), 0)$ if $x \in S,(\sigma(x), 1)$ otherwise, and 
$f(x, 1)=(\tau(x), 1)$ if $x \in S,(\tau(x), 0)$ otherwise: then $g$ is bijective because, w.r.t. $f$, the roles of $(x, 0)$ and $(y, 1)$ are permuted exactly when $\sigma(x)=\tau(y)$, i.e., $y=\tau^{-1} \sigma(x)$. For it to be hereditarily bijective (and not merely bijective), one needs to take $S$ stable under the actions of $\left(\tau \uparrow_{\kappa}\right)^{-1} \sigma \uparrow_{\kappa}$ for any subcube $\kappa$.

These stable sets $S$ may further be characterized as follows, through the orthogonality relation defined in Section 4. Consider the binary relation $x \smile y$ on $\mathbb{B}^{n}$ defined by $[x, y] \perp[\sigma(x), \tau(y)]$ : by Lemma 4 , if $x+y=e_{I}$ and $\sigma(x)+\tau(y)=$ $e_{J}, x \smile y$ is equivalently defined by $I \cap J=\varnothing$. This relation generates an equivalence relation, whose equivalence classes are the required sets.

\section{References}

1. C. Chaouiya and E. Remy. Logical modelling of regulatory networks, methods and applications. Bulletin of Mathematical Biology, 75:891-895, 2013.

2. H. de Jong. Modeling and simulation of genetic regulatory systems: A literature review. Journal of Computational Biology, 9(1):67-103, 2002.

3. J. Demongeot, A. Elena, and S. Sené. Robustness in regulatory networks: a multidisciplinary approach. Acta Biotheoretica, 56(1-2):27-49, 2008.

4. E. Goles and L. Salinas. Comparison between parallel and serial dynamics of Boolean networks. Theoretical Computer Science, 396:247-253, 2008.

5. S. A. Kauffman. Metabolic stability and epigenesis in randomly constructed genetic nets. Journal of Theoretical Biology, 22:437-467, 1969.

6. S. A. Kauffman. The origins of order: Self-organization and selection in evolution. Oxford University Press, 1993.

7. E. Remy, P. Ruet, and D. Thieffry. Graphic requirements for multistability and attractive cycles in a Boolean dynamical framework. Advances in Applied Mathematics, 41(3):335-350, 2008.

8. A. Richard. A fixed point theorem for Boolean networks expressed in terms of forbidden subnetworks. In Cellular Automata and Discrete Complex Systems, DMTCS Proceedings, 2012.

9. A. Richard and P. Ruet. From kernels in directed graphs to fixed points and negative cycles in Boolean networks. Discrete Applied Math., 161:1106-1117, 2013.

10. P. Ruet. Local cycles and dynamical properties of Boolean networks. To appear in Mathematical Structures in Computer Science, 2014.

11. M.-H. Shih and J.-L. Dong. A combinatorial analogue of the Jacobian problem in automata networks. Advances in Applied Mathematics, 34(1):30-46, 2005.

12. E. H. Snoussi. Qualitative dynamics of piecewise-linear differential equations: a discrete mapping approach. Dynamics and Stability of Systems, 4(3-4), 1989.

13. R. Thomas. Boolean formalization of genetic control circuits. Journal of Theoretical Biology, 42:563-585, 1973

14. R. Thomas. On the relation between the logical structure of systems and their ability to generate multiple steady states and sustained oscillations. In Series in Synergetics, volume 9, pages 180-193. Springer, 1981.

15. R. Thomas. Regulatory networks seen as asynchronous automata: a logical description. Journal of Theoretical Biology, 153:1-23, 1991.

16. R. Thomas and M. Kaufman. Multistationarity, the basis of cell differentiation and memory. I. Structural conditions of multistationarity and other non-trivial behaviour. Chaos, 11:170-179, 2001.

17. J. von Neumann. Theory of self-reproducing automata. University of Illinois Press, 1966. 